\title{
Patrones de infestación por insectos xilófagos en renovales de Nothofagus obliqua Mirb. y Nothofagus dombeyi (Mirb.) Oerst. (Fagales: Nothofagaceae)
}

\author{
Infestation patterns of xylophagous insects in second growth stands of Nothofagus obliqua \\ Mirb. and Nothofagus dombeyi (Mirb.) Oerst. (Fagales: Nothofagaceae)
}

LORENA H. SUÁREZ ${ }^{1, *}$ ANGÉLICA V. AGUILAR² \& WILFREDO L. GONZÁLES ${ }^{1}$

\author{
${ }^{1}$ ECOBIOSIS, Departamento de Botánica, Universidad de Concepción. \\ Casilla 160-C, Concepción, Chile \\ ${ }^{2}$ Instituto de Silvicultura, Facultad de Ciencias Forestales, Universidad Austral de Chile, Valdivia, Chile. \\ *Autor para correspondencia; e-mail: 1suarez@udec.cl
}

\begin{abstract}
RESUMEN
Evaluamos los patrones de infestación por insectos xilófagos en renovales de Nothofagus obliqua y Nothofagus dombeyi en las provincias de Valdivia, Osorno y Llanquihue (Chile). Realizamos los análisis a dos escalas: en primer lugar se consideraron las variables diámetro a la altura del pecho (DAP) y clase de copa del árbol hospedante (CC) como predictores de daño por xilófagos a nivel del árbol. En segundo lugar se consideraron las variables densidad arbórea total, densidad de hospedante, número y cobertura de especies (estas últimas agrupadas en los estratos arbóreo, arbustivo, de herbáceas y de epífitas), y su asociación con el porcentaje de infestación a nivel de rodal. El área de estudio presentó una infestación promedio de 9,35\% en $N$. obliqua y $15,89 \%$ en $N$. dombeyi. A nivel individual tanto el DAP como la CC se relacionan positivamente con la presencia de daño, sin embargo el DAP resultó mejor predictor tanto para $N$. obliqua como para $N$. dombeyi, aumentando la probabilidad de encontrar un árbol dañado con el aumento del DAP. A nivel de rodal se encontró que las variables estudiadas no se relacionan con la infestación en $N$. obliqua, mientras que en $N$. dombeyi renovales con mayor número de especies en el estrato arbustivo y/o con mayor densidad de rodal y hospedante presentaron menores niveles de infestación.
\end{abstract}

Palabras clave: herbivoría, insectos xilófagos, insectos barrenadores de xilema, Nothofagus obliqua, Nothofagus dombeyi.

\begin{abstract}
Infestation patterns of xylophagous insects were evaluated in second growth stands of Nothofagus obliqua and Nothofagus dombeyi in Valdivia, Osorno and Llanquihue provinces of Chile. The analysis addressed two scales. First, at the individual level, diameter at breast height (DBH) and canopy class (CC) of the host tree were used as predictor traits of xylophagous damage. Second, at the stand level, variables such as total tree density, host density, species richness and cover (arboreal, shrubby, herbaceous and epiphytic strata) and their asociation with infestation average were considered. The infestation mean was $9.35 \%$ for $N$. obliqua and $15.89 \%$ for N. dombeyi. At individual level both DBH and CC were positively associated with damage presence. However, the best predictor of tree infestation was DBH in both $N$. dombeyi and $N$. obliqua, greater DBH increasing the probability to find an infested tree. At the stand level, no relationship was found between insect infestation and evaluated traits in $N$. obliqua, whereas $N$. dombeyi stands with high species richness in the shrubby stratum and/or with higher total tree density and host density showed the lowest infestation levels.
\end{abstract}

Key words: herbivory, xylophagous insect, wood-boring insect, Nothofagus obliqua, Nothofagus dombeyi.

\section{INTRODUCCIÓN}

Un tipo de herbivoría frecuente en árboles es la provocada por insectos barrenadores (Hanks 1999), los que se caracterizan por la construc- ción de galerías en tejidos del xilema, floema o corteza mientras se alimentan durante el estado larval. Tanto la ocurrencia como la intensidad del daño producido dependen de factores propios del hospedante (tamaño, condición de vi- 
gor/estrés y presencia de componentes químicos atractivos y defensivos [Price 1997, Hanks 1999]) y factores ambientales (densidad total de plantas, densidad de hospedante, complejidad en la composición, estructura florística y variables climáticas), los que son comunes a una población. Estos factores pueden afectar los niveles de daño tanto a través de la influencia en el éxito de establecimiento y posterior desempeño del insecto, como a través del efecto sobre el desarrollo individual del hospedante, determinando la susceptibilidad al daño.

Particularmente, la infestación por barrenadores ha sido positivamente asociada con el diámetro del árbol hospedante (Zhang et al. 1993, Cabrera 1994, Díaz 1999, Reid \& Robb 1999, Carrasco 2000, Manqui 2001, Jactel et al. 2002). Los posibles mecanismos causales detrás de este patrón son variados y se pueden agrupar en dos: (1) aquellos asociados a la capacidad de detección por parte del insecto, y (2) los asociados con su desempeño, particularmente con la sobrevivencia y éxito reproductivo. En el primer grupo están las características químicas del hospedante. Se han encontrado asociaciones positivas entre el diámetro de los árboles hospedantes y la presencia de compuestos químicos atractivos para el insecto dañador (Jactel et al. 2002). Por otra parte, Lawton (1983) sugirió que árboles de mayor tamaño son más fáciles de descubrir por su apariencia que los más pequeños ("per se size hypothesis"). Mayores densidades del hospedante podrían facilitar el encuentro por parte del insecto y/o permitir un mejor desempeño debido a la concentración del recurso (Root 1973). Dentro del segundo grupo, el tipo de corteza del hospedante puede ser un factor relevante. En algunas especies, diámetros mayores están asociados a cortezas más gruesas y rugosas (Kruuse 1981; Zhang et al. 1993), las que ofrecerían mejores condiciones para la oviposición (Hanks 1999). Por otra parte, la diversidad florística podría afectar tanto el éxito de establecimiento como la sobrevivencia del insecto. Algunos autores sostienen que la herbivoría de insectos que tienden a ser más específicos en cuanto a sus hospedantes puede disminuir en condiciones de mayor diversidad (Lawton 1983, Andow 1991, Altieri 1992, Landis et al. 2000), pues en estos sistemas un mayor número de especies vegetales sostiene una mayor diversidad de herbívoros, los que a su vez sostienen un mayor número de parásitos y depredadores (enemigos naturales).
En renovales del género Nothofagus, particularmente en renovales de $N$. obliqua y $N$. dombeyi, se ha reportado daño por insectos xilófagos nativos (Cameron \& Peña 1982, Cabrera 1994, Grandon 1996, Aguilar et al. 1997), siendo Nothofagus obliqua atacado principalmente por el coleóptero Holopterus chilensis (Blanch), llamado comúnmente "gusanera del roble", y Nothofagus dombeyi por Cheloderus childreni (Gray), conocido como "coleóptero de la luma" (Peña 1974, Cameron \& Peña 1982, Carrillo \& Cerda 1987, Grandon 1996, Aguilar et al. 1997). Ambos insectos pertenecen a la familia Cerambycidae. Algunos autores han sugerido que son especialistas (Kruuse 1981, Aguilar et al. 1997; pero véase Cameron \& Peña 1982, Grandon 1996) y que atacan a su hospedante vivo (Cameron \& Peña 1982). El daño producido por las larvas al construir galerías dentro del tronco durante su alimentación puede llegar a destruir casi por completo los primeros metros del fuste desde el suelo (Carrillo \& Cerda 1987, Cabrera 1994, Aguilar et al. 1997, Díaz 1999).

El objetivo del presente trabajo fue evaluar los patrones de daño en renovales de $N$. obliqua y $N$. dombeyi ocasionado por insectos barrenadores de xilema en la Décima Región de Chile, para lo cual se determinó el grado de infestación de ambas especies y se realizaron los siguientes análisis: (1) a escala individual se relacionó el daño a nivel de árbol (presencia o ausencia) con el diámetro a la altura del pecho (DAP) y clase de copa (CC) del hospedante, y (2) a escala poblacional (intensidad de daño a nivel poblacional) se estableció la relación entre el porcentaje de infestación con las variables densidad total de rodal, densidad de hospedante y número y cobertura de las especies vegetales asociadas, estas últimas como variables indicadoras de riqueza y abundancia florística.

\section{MATERIALES Y MÉTODOS}

\section{Renovales de Nothofagus}

Las formaciones de renovales de $N$. obliqua, $N$. dombeyi y mixtas pertenecen, según la tipología forestal desarrollada por Donoso (1981), al tipo roble-raulí-coigüe, subtipo renoval y bosque puro secundario. Se distribuyen desde 
los 36ํㅜㅇ' S hasta los 41으 S (Donoso 1994). Se trata de comunidades vegetales discretas, coetáneas, de gran variación estructural, originadas a partir de fenómenos naturales o antrópicos de carácter catastrófico (Puente et al. 1979, Donoso 1981). Dependiendo de la edad del bosque, su composición y localización se pueden encontrar rodales con DAP promedios entre $\operatorname{los} 5$ y $\operatorname{los} 50 \mathrm{~cm}$ y densidades en un rango de 600 a 5.000 árboles ha-1 (Donoso 1981). En los renovales aparecen especies arbóreas sombra-tolerantes que formaron parte del bosque anterior y que se encuentran generalmente en los estratos de dosel intermedios o suprimidos. Algunas de ellas son: Aextoxicon punctatum (Aextoxicaecae), Gevuina avellana (Proteaceae), Luma apiculata (Myrtaceae), Weinmannia trichosperma (Cunoniaceae), Dasyphyllum diacanthoides (Compositae), Laurelia sempervirens (Monimiaceae), Amomyrtus meli (Myrtaceae), Amomyrtus luma (Myrtaceae) y Persea lingue (Lauraceae).

\section{Xilófagos asociados}

Holopterus chilensis (Blanch). Esta especie se distribuye desde la provincia de Arauco hasta Osorno, comuna de Río Negro (Kruuse 1981), coincidiendo en toda su distribución con $N$. obliqua. El adulto es de color café claro y presenta dimorfismo sexual en tamaño y longitud de antenas. Las hembras alcanzan tamaños de hasta $47 \mathrm{~mm}$ de longitud y $11 \mathrm{~mm}$ de ancho y sus antenas alcanzan $75 \%$ de su longitud total. Los machos alcanzan $42 \mathrm{~mm}$ de longitud y $8 \mathrm{~mm}$ de ancho y sus antenas sobrepasan la longitud corporal (Kruuse 1981). El insecto posee hábitos nocturnos. Poco antes de la dispersión de los machos, estos son atraídos por feromonas producidas por la hembra (Gara et al. 1978), la que una vez fecundada, deposita los huevos aislados sobre la corteza, principalmente en la base de los árboles hasta una altura de $1 \mathrm{~m}$. Los huevos miden en promedio 6,22 $\mathrm{mm}$ de largo y 3,01 $\mathrm{mm}$ de ancho (Kruuse 1981) y son de color amarillo opaco. La larva es blanca y puede medir $55 \mathrm{~mm}$ de largo (Kruuse 1981). H. chilensis construye su cámara pupal con tapones de viruta en el orificio de emergencia del adulto. Aunque el ciclo de vida no se ha determinado, se estima que es superior a dos años. Los huevos se pueden en- contrar entre los meses de diciembre a febrero, larvas durante todo el año, pupas entre julio y diciembre y adultos entre diciembre y enero (Kruuse 1981). No existe información sobre enemigos naturales. El daño es provocado cuando la larva construye galerías en el fuste mientras se alimenta y puede ser reconocido por la presencia de exudaciones de savia, orificios de emergencia y evacuación de aserrín, el que tiene una textura granular característica (Kruuse 1981). Aunque las galerías se concentran en los primeros metros, Díaz (1999) encontró daño interno hasta 4,6 m de altura.

Cheloderus childreni (Gray). Se distribuye desde la provincia de Curicó hasta Aysén (Cameron \& Peña 1981). El adulto es de color verde metálico en el tórax y abdomen, rojo verdoso en los élitros y azul en las patas. Alcanza tamaños de hasta $39 \mathrm{~mm}$ de longitud y $14 \mathrm{~mm}$ de ancho (Cameron \& Real 1974), siendo las hembras más grandes que los machos. Posee hábitos diurnos y tanto hembras como machos vuelan, aunque la hembra lo hace deficientemente (Gara et al. 1978). La cópula tiene lugar en la corteza de árboles. Los machos son atraídos por feromonas producidas por la hembra, la que una vez fecundada oviposita sobre la corteza, principalmente en la base de los árboles hasta una altura de $3 \mathrm{~m}$. Los huevos son blanco-amarillentos, miden de 4,7 a $5 \mathrm{~mm}$ de largo y 2,7 a $3,2 \mathrm{~mm}$ de ancho y son recubiertos con material que la hembra raspa de la corteza al momento de la ovipostura (Cameron \& Real 1974). Al emerger la larva del huevo, esta abre un agujero entrando directamente al xilema atravesando corteza y cambium. La larva es blanca y puede medir $50 \mathrm{~mm}$ de largo (Cameron \& Real 1974). Cuando termina su desarrollo, la larva cava un orificio de salida que luego cierra con un tapón de viruta formando la cámara pupal. El ciclo de vida se ha estimado en cinco a seis años. Los huevos se pueden encontrar entre los meses de noviembre a agosto, larvas durante todo el año, pupas entre septiembre y febrero, adultos entre noviembre y abril (Cameron \& Real 1974). Entre los depredadores conocidos están Campephilus maguellanicus (Picidae), o pájaro carpintero, que utiliza los tapones de aserrín como señal para encontrar la pupa (Cameron \& Real 1974), y Milvago chimango (Falconidae), o tiuque, depredador del estado adulto. 
El daño producido por $C$. childreni puede ser reconocido por el tipo de aserrín que produce la larva mientras se alimenta. Este es blanco-amarillento y se encuentra tanto en la corteza como acumulado en la base del árbol.

Una completa recopilación sobre los antecedentes de historia natural de ambos insectos se encuentra en Baldini \& Pancel (2002).

\section{Área de estudio}

El área de estudio se estableció en renovales dominados por las especies $N$. dombeyi o $N$. obliqua ubicados en las provincias de Valdivia, Osorno y Llanquihue, Décima Región de Chile (Fig. 1). La colecta de datos se efectuó en los meses de enero, febrero y marzo de 2002.

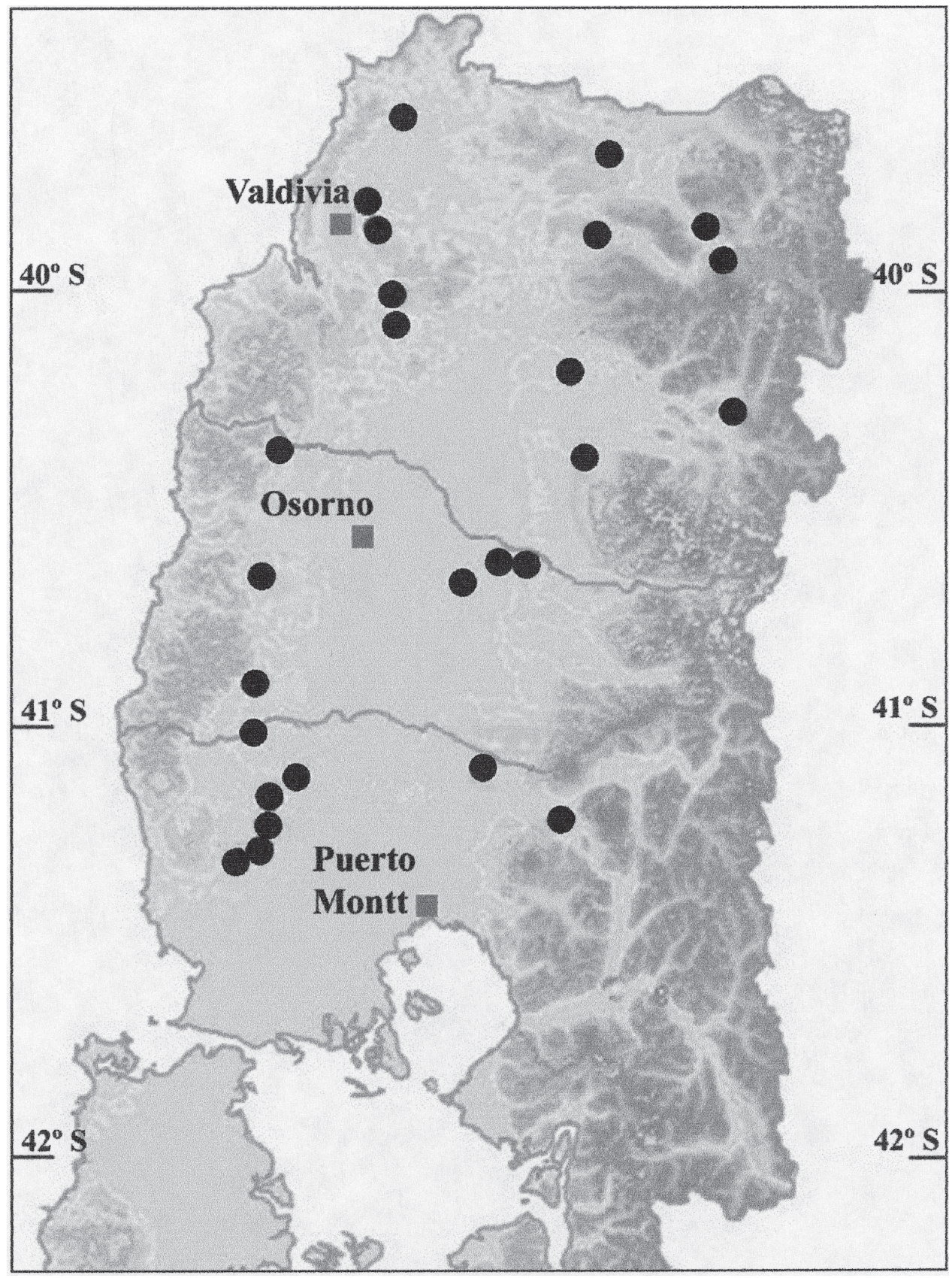

Fig.1: Ubicación geográfica del área de estudio. Parcelas señaladas con círculos $(\bullet)$. Geographical location of the study area. Plots are marked with circles $(\bullet)$. 


\section{Establecimiento de parcelas y variables} medidas

Se hizo un inventario en 11 parcelas dominadas por $N$. dombeyi y 17 por $N$. obliqua. Las parcelas de forma rectangular, con 250 ó 500 $\mathrm{m}^{2}$ de superficie (dependiendo de la densidad de fustes) albergan un total de 1.783 árboles de Nothofagus, de los cuales 544 son de $N$. dombeyi $\left(49,0 \pm 10,1\right.$ árboles parcela ${ }^{-1}$; media $\pm \mathrm{EE})$ y 1.239 de $N$. obliqua $(72,3 \pm 7,7$ árboles parcela ${ }^{-1}$ ).

Se registraron las siguientes variables individuales: especie; diámetro a la altura del pecho (DAP); clase de copa (CC: posición de los individuos del dosel arbóreo según Kraft (descrito en Donoso 1994); categorías: dominante: aquellos cuyas copas sobresalen ligeramente del dosel general; codominante: árboles poco más bajos que los dominantes, que constituyen parte del dosel superior general; intermedio: árboles que se encuentran claramente en posición subdominada, en general fuertemente oprimidos por individuos dominantes y codominantes; suprimido: ubicados totalmente bajo el dosel, no reciben luz solar directa e infestación (presencia o ausencia de daño según la presencia de signos externos como orificios de entrada, salida y evacuación de aserrín). También se registraron las siguientes variables del rodal: densidad arbórea total (número ha-1), densidad de hospedante (número ha-1 ${ }^{-1}$, número (número parcela ${ }^{-1}$ ) y cobertura $(\%)$ de especies.

Para determinar el número y cobertura de especies se utilizaron 28 parcelas de $500 \mathrm{~m}^{2}$ en las cuales se realizó censos de vegetación en cuatro estratos definidos en base a la distribución vertical de las especies en el bosque. En cada estrato se registraron las especies presentes y se estimó la cobertura absoluta de cada una de ellas en porcentaje de superficie. Estratos: arbóreo: especies arbóreas adultas de CC dominante, codominante e intermedia; arbustivo: especies arbustivas y arbóreas jóvenes (brinzales y latizales) con menos de $5 \mathrm{~cm}$ de DAP, y árboles adultos de CC suprimida; herbáceo: especies arbóreas en estado de regeneración, hierbas, helechos y arbustos (altura total menor a $50 \mathrm{~cm}$ ); de epífitas y trepadoras: comprende especies creciendo sobre fustes y ramaje de otras especies, inclusive aquellas que enraízan en el suelo.

\section{Análisis de datos}

A nivel individual la variable respuesta infestación se trabajó como variable dicotómica $1 / 0$, donde $1=$ presencia de daño y $0=$ ausencia de daño. Los árboles se agruparon según infestación y se comparó entre grupos los promedios de DAP, mediante una prueba de t de Student, y la CC mediante la prueba U de Mann-Whitney. Adicionalmente, ambos factores se evaluaron en conjunto a través de un modelo lineal mixto generalizado (GLIMMIX macro, SAS Institute 1997), utilizando una función logit y una distribución binomial del error. Las variables consideradas en este modelo fueron: parcela (bloque), DAP y CC (estas últimas anidadas en parcela), como variables aleatorias y especie como factor fijo.

A nivel de rodal se calculó el porcentaje de infestación para cada especie, considerando el número de árboles dañados en porcentaje del total. Se evaluó la correlación (Pearson) entre el porcentaje de infestación y las variables densidad total de rodal, densidad de hospedante, número y cobertura de especies por estrato. Adicionalmente se realizó un análisis de regresión múltiple ("forward stepwise") seleccionando las mejores variables predictoras de infestación.

\section{RESULTADOS}

\section{Análisis de nivel individual}

Los promedios de DAP en árboles infestados fueron significativamente mayores en comparación con los árboles sanos (Fig. 2) en ambas especies estudiadas (prueba $\mathrm{t}$ de Student, $\mathrm{t}_{544}=$ 6,5; $\mathrm{P}<0,00001$ para N. dombeyi $\mathrm{t}_{1237}=2,4$; $\mathrm{P}<0,05$ para $N$. obliqua). En relación a la CC, se encontró que árboles infestados se ubican más en las posiciones dominante y codominante que en las posiciones intermedia y suprimida tanto en $N$. dombeyi (prueba U de MannWhitney, $\left.\mathrm{Z}_{544}=3,58 ; \mathrm{P}<0,001\right)$ como en $N$. obliqua $\left(\mathrm{Z}_{1237}=2,40 ; \mathrm{P}<0,05\right)$. El análisis múltiple GLIMMIX (Tabla 1) muestra que el diámetro afecta la probabilidad de daño y que $N$. dombeyi y $N$. obliqua son dañados igualmente. El efecto de la clase de copa no es significativo en esta escala de análisis. No se detectó efecto de parcela. 


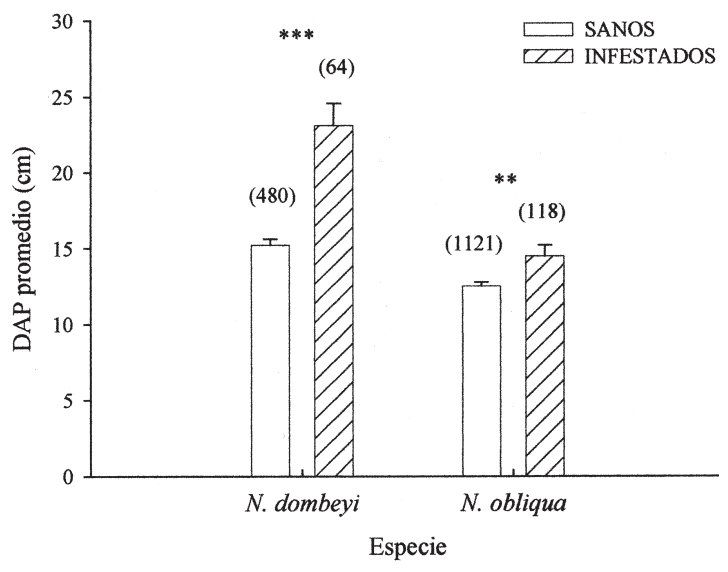

Fig.2: Promedio del diámetro a la altura del pecho (DAP) para árboles sanos e infestados. Tamaño muestral señalado entre paréntesis. Barras de error indican ${ }^{1} \mathrm{EE}$; (*) $\mathrm{P}<0,05$; (**) $\mathrm{P}<$ 0,$005 ;(* * *) \mathrm{P}<0,0001$ (prueba t de Student).

Average diameter at breast height (DBH) in healthy and infested trees. Sample size indicated between brackets. Bars $\operatorname{are}^{1} \mathrm{SE}$; (*) $\mathrm{P}<0,05 ;(* *) \mathrm{P}<0,005 ;$ (***) $\mathrm{P}<$ 0,0001 (Student t-test).

\section{TABLA 1}

Análisis de la infestación de Nothofagus dombeyi y $N$. obliqua, usando un modelo lineal mixto generalizado; DAP = diámetro a la altura del pecho; $\mathrm{CC}=$ clase de copa

Analysis of infestation of Nothofagus dombeyi and N. obliqua using a generalized linear mixed model;

$\mathrm{DAP}=$ diameter at breast height $\mathrm{CC}=$ canopy class

\begin{tabular}{lccc}
\hline Variable & Estima & Error estándar & Valor de Z \\
\hline DAP (parcela) & 0,010 & 0,004 & $2,31^{*}$ \\
CC (parcela) & 0,194 & 0,191 & $1,02^{\mathrm{NS}}$ \\
Parcela & 1,583 & 0,878 & $1,80^{\mathrm{NS}}$ \\
Factor fijo & $\mathrm{GLN}^{1}$ & $\mathrm{GLD}^{2}$ & $\mathrm{~F}$ \\
Especie & 1 & 1690 & $0,02^{\mathrm{NS}}$ \\
\hline
\end{tabular}

$(*)=\mathrm{P}<0,05$; obtenido mediante prueba de razón de verosimilitud; $(\mathrm{NS})=\sin$ diferencias significativas; ${ }^{1} \mathrm{GLN}=$ grados de libertad nominador; ${ }^{2} \mathrm{GLD}=$ grados de libertad denominador

\section{Análisis a nivel de rodal}

Se calculó una infestación de 9,35\% para $N$. obliqua y $15,89 \%$ para $N$. dombeyi (Tabla 2). La Tabla 2 muestra los estadígrafos de dispersión central para las variables de rodal. A través del análisis de correlación se encontró que la infestación en $N$. dombeyi se relaciona negativamente con la densidad total de rodal (análi- sis de correlación de Pearson; $\mathrm{R}=-0,73 ; \mathrm{P}<$ $0,025)$ y de hospedante $(\mathrm{R}=-0,67 ; \mathrm{P}<0,02)$ y con el número de especies en el estrato de arbustos $(\mathrm{R}=-0,65 ; \mathrm{P}<0,05)$. La infestación en $N$. obliqua no mostró asociación con las variables medidas.

Según el análisis múltiple, el número de especies del estrato arbustivo mostró una relación negativa con la infestación de $N$. dombeyi, mientras que la densidad de rodal no (Tabla $3 \mathrm{~A})$. En rodales de $N$. obliqua no se encontró relación de la infestación con las variables estudiadas (Tabla 3B).

\section{DISCUSIÓN}

En ambas especies de Nothofagus los diámetros de los individuos infestados fueron mayores que los de los individuos sanos (Fig. 2). Este patrón puede deberse a que individuos de mayor diámetro presentarían mejores condiciones para la atracción y desarrollo del insecto probablemente asociadas a dos características: (a) mayor abundancia relativa de madera duraminizada, y (b) corteza más gruesa y rugosa. En el duramen se encuentra mayor cantidad de compuestos fenólicos y/o volátiles, sustancias que se han asociado anteriormente a la infestación por distintas especies de insectos barrenadores (Dunn et al. 1990, Hanks et al. 1993, Zhang et al. 1999) y reconocidas como compuestos atractores químicos naturales que facilitan el encuentro del árbol hospedante (Linsley 1959, Kleinhentz et al. 1999, Barata et al. 2002). Insectos que se alimentan en los árboles pueden ubicar a su hospedante a través de una combinación de señales visuales y olfativas (Byers 1989). Por otra parte, una relación positiva entre el grosor de corteza y el éxito reproductivo ha sido reportada para otros cerambícidos barrenadores (Zhang et al. 1993). En Holopterus chilensis, Kruuse (1981) encontró que las hembras prefieren ovipositar en los lugares más rugosos de la corteza de $N$. obliqua, tanteando con las antenas la superficie de la corteza y ubicando el ovipositor en las estrías longitudinales entre placas. La rugosidad y grosor de la corteza estarían entregando al insecto mejores condiciones para la ovipostura, pues los huevos quedan más protegidos entre las capas o placas de corteza. Esto puede disminuir la probabilidad de ataque por depredadores y por 
otro lado puede representar un microambiente propicio para la sobrevivencia de huevos y primeros estadios larvales (Lawton 1983). Muchos autores concuerdan en que las condiciones ofrecidas por los árboles para la ovipostura del insecto conforman un factor de gran importancia a la hora de la elección del hospedante (Lawton 1983, Thompson 1988, Hanks et al.
1993, Hanks 1999). Esto es particularmente relevante en el caso de cerambícidos barrenadores de xilema, ya que en muchos casos la larva es incapaz de cambiarse de un hospedero a otro durante su desarrollo (Hanks 1999). En H. chilensis, insecto asociado a la infestación en $N$. obliqua, Kruuse (1981) reportó que todo el desarrollo larval ocurre en el mismo hospedante.

TABLA 2

Estadística descriptiva para los atributos evaluados a nivel de parcela; $\mathrm{N}=$ número de especies; $\mathrm{E}=$ estrato $\mathrm{C}=$ cobertura; $\mathrm{n}=$ número de parcelas

Descriptive statistics to the plot level traits evaluated; $\mathrm{N}=$ species number; $\mathrm{E}=$ stratum; $\mathrm{C}=$ cover; $\mathrm{n}=$ number of plots

\begin{tabular}{|c|c|c|c|c|}
\hline \multirow[t]{2}{*}{ Atributo } & \multicolumn{2}{|c|}{$N$. dombeyi $(\mathrm{n}=17)$} & \multicolumn{2}{|c|}{ N. obliqua $(\mathrm{n}=11)$} \\
\hline & Media & $\mathrm{EE}^{1}$ & Media & $\mathrm{EE}$ \\
\hline $\mathrm{N}$ total $\left(\mathrm{N} \mathrm{ha}^{-1}\right)$ & 23,53 & 1,89 & 24,91 & 1,84 \\
\hline $\mathrm{N}$ E arbóreo $\left(\mathrm{N} \mathrm{ha}^{-1}\right)$ & 4,29 & 0,65 & 6,45 & 1,17 \\
\hline $\mathrm{N}$ E arbustivo $\left(\mathrm{N} \mathrm{ha}^{-1}\right)$ & 6,76 & 0,75 & 7,45 & 0,85 \\
\hline $\mathrm{N}$ E epífitas $\left(\mathrm{N} \mathrm{ha}^{-1}\right)$ & 5,76 & 0,75 & 7,45 & 0,78 \\
\hline N E herbáceas $\left(\mathrm{N} \mathrm{ha}^{-1}\right)$ & 7,59 & 1,11 & 3,64 & 0,83 \\
\hline C E arbóreo (\%) & 105,29 & 12,49 & 139,18 & 18,62 \\
\hline $\mathrm{C}$ E arbustivo (\%) & 73,76 & 15,49 & 86,64 & 20,40 \\
\hline C E epífitas (\%) & 14,00 & 4,12 & 18,91 & 7,30 \\
\hline C E herbáceas (\%) & 49,35 & 14,93 & 4,36 & 1,03 \\
\hline DAP promedio $(\mathrm{cm})$ & 15,07 & 1,90 & 18,86 & 2,61 \\
\hline Densidad de rodal $\left(\mathrm{N} \mathrm{ha}^{-1}\right)$ & 2875,29 & 383,09 & 2614,55 & 329,89 \\
\hline Densidad de hospedante $\left(\mathrm{N} \mathrm{ha}^{-1}\right)$ & 2275,29 & 351,45 & 1170,91 & 280,43 \\
\hline Infestación (\%) & 9,35 & 3,16 & 15,89 & 4,58 \\
\hline
\end{tabular}

${ }^{1} \mathrm{EE}=1$ error estándar

Análisis de regresión múltiple ("forward stepwise”), para establecer la relación entre el porcentaje de infestación y las variables de estudio

Forward stepwise multiple regression analysis to establish the relationship between infestation percentage and study traits

\begin{tabular}{lcc}
\hline Especie y parámetros de la regresión & Coeficiente (EE) & Valor de $\mathrm{t}$ \\
\hline (A) Nothofagus dombeyi & & \\
$\mathrm{F}_{3,7}=10,830 ; \mathrm{P}<0,0050 ; \mathrm{R}^{2}=0,74$ & $-3,965(0,954)$ & $-4,15^{* *}$ \\
Número de especies estrato arbustivo $\left(\mathrm{N} \mathrm{ha}^{-1}\right)$ & $-2,169(1,173)$ & $-1,84^{\mathrm{NS}}$ \\
Número de especies estrato de epífitas $\left(\mathrm{N} \mathrm{ha}^{-1}\right)$ & $-0,005(0,003)$ & $-1,96^{\mathrm{NS}}$ \\
Densidad de rodal $\left(\mathrm{N} \mathrm{ha}^{-1}\right)$ & & \\
(B) Nothofagus oblicua & $0,072(0,049)$ & $1,48^{\mathrm{NS}}$ \\
$\mathrm{F}_{2,14}=1,831 ; \mathrm{P}<0,1965 ; \mathrm{R}^{2}=0,20$ & $-5,148(3,847)$ & $-1,33^{\mathrm{NS}}$ \\
Cobertura de especies estrato arbustivo $(\%)$ & \\
Cobertura de especies estrato de epífitas $(\%)$ & \\
\hline
\end{tabular}

$(* *)=\mathrm{P}<0,005 ;(\mathrm{NS})=$ sin diferencias significativas 
Otro factor asociado al DAP es el posible efecto acumulativo de infestaciones pasadas tanto del mismo insecto o de otros agentes patógenos como hongos, virus o bacterias. Si bien al medir la presencia de daño a partir de signos externos frescos como presencia de aserrín y orificios de emergencia/evacuación no se considera daño acumulado (sino solo aquel provocado por las larvas de una temporada), árboles más viejos pueden presentar mayor intensidad de daño no detectable externamente, debido a que han estado expuestos mayor tiempo a los agentes patógenos. Pequeñas diferencias de edad dentro de un renoval pueden estar asociadas a diferencias de tamaño en diámetro, por lo que un posible efecto de la historia de daño de un individuo sobre la infestación actual, puede ser particularmente importante en aquellos árboles de mayor DAP. Estudios previos en barrenadores han reportado que árboles con daño anterior del mismo insecto (otras temporadas) y de otros son más susceptibles a un nuevo ataque que árboles sanos (Elliott et al. 1987). En el caso de los renovales estudiados, la probabilidad de reinfestación puede ser alta ya que las hembras, condicionadas por un vuelo deficiente en el caso de Ch. childreni, podrían presentar baja dispersión.

La relación existente entre la infestación y $\mathrm{CC}$ puede deberse a que árboles más grandes son más fáciles de descubrir y colonizar por sus potenciales huéspedes que los más pequeños (Lawton 1983). Por otro lado, otros trabajos han reportado que la altura de los árboles se correlaciona positivamente con el DAP en renovales de Nothofagus (De Camino \& Drake 1977). En este estudio se encontró que los promedios de diámetro de los árboles dominantes y codominantes (mayor altura) son significativamente mayores que en las otras dos posiciones (L.H. Suárez resultados no publicados), de manera que la asociación entre CC e infestación podría estar sujeta a un efecto enmascarado del diámetro. El análisis múltiple (Tabla 1) mostró que el mejor predictor de daño es el DAP, aun cuando ambas variables se asocian positivamente a la infestación como lo muestran los análisis individuales. Otros factores pueden contribuir a explicar la relación entre infestación y tamaño, como por ejemplo la condición de vigor/estrés, pues muchos insectos atacan preferentemente hospedantes de una condición particular (Dunn et al. 1986, Hanks 1999, Inbar et al. 2001).
Con respecto a la densidad arbórea y según el análisis de correlación, árboles de $N$. dombe$y i$ son más infestados en rodales cuya densidad total es menor, aun cuando esta relación es marginal según el análisis múltiple. Una posible explicación se relaciona con el comportamiento del insecto en la época de apareamiento. Aunque se sabe que la atracción sexual de estos insectos se realiza principalmente por medio de feromonas producidas por la hembra (Gara et al. 1978), los mismos autores observaron que en la época de apareamiento de Ch. childreni (asociado a la infestación en $N$. dombeyi), las hembras realizaron despliegues muy llamativos, pues al incidir los rayos del sol en los colores metálicos del tórax y élitros junto con ciertos movimientos provocados por el insecto, se producían destellos lumínicos que se consideraron como un probable método de atracción. En este sentido, rodales menos densos podrían facilitar el recibimiento de estas señales visuales y el consiguiente encuentro de la hembra por parte del macho para el apareamiento. Esto no sería importante en el caso de $H$. chilensis dados sus hábitos nocturnos.

La riqueza y abundancia florística (en este caso expresada como número y cobertura de especies) afecta al daño provocado en $N$. dombeyi, concordando con muchos autores en que a mayor diversidad y complejidad en la estructura del hábitat, la herbivoría tiende a ser menor (Lawton 1983, McCann \& Harman 1990, Andow 1991, Coll \& Bottrell 1994, Tonhasca \& Byrne 1994, Humphrey et al. 1999, Zhang et al. 1999, Landis et al. 2000). El aumento de especies y de su abundancia trae consigo el aumento de señales químicas que pueden dificultar el encuentro del hospedante e incluso inhibir el efecto de sustancias atractoras que el insecto sea capaz de percibir, lo que puede ser particularmente relevante en este caso considerando que el patrón de daño asociado a estos insectos se caracteriza por oviposturas y entrada de larvas bajo $1,5 \mathrm{~m}$ de altura en el fuste (Kruuse 1981, Cabrera 1994, Díaz 1999). Un ejemplo de interferencia química es reportado por Zhang et al. (1999) para un xilófago de especies de abetos, mostrando que la respuesta del insecto a señales atractoras llegó a ser inhibida en presencia de compuestos volátiles emitidos por especies latifoliadas acompañantes al hospedante. Por otro lado, una mayor complejidad del ecosistema en cuanto a la riqueza florística, puede soportar una mayor abundancia de 
enemigos naturales generalistas (Lawton 1983, Andow 1991, Landis 2000). Así, el nivel de depredación por el generalista Milvago chimango sobre $C h$. childreni puede ser mayor en hábitat con mayor riqueza florística. A pesar de que la hipótesis referida a la diversidad florística puede explicar el patrón encontrado en $N$. dombeyi, este no ocurre en $N$. obliqua. Una hipótesis que se podría considerar es que $C h$. childreni, debido a su vistosidad, puede ser más depredado comparativamente con $H$. chilensis, aun en ambientes más diversos, dada la apariencia críptica de este último.

Sin duda este trabajo es una aproximación inicial que muestra un patrón. Para ser concluyentes en cuanto a los mecanismos detrás de las asociaciones establecidas se requiere generar información a partir de aproximaciones experimentales, tanto en laboratorio como en el campo. Son necesarios trabajos futuros orientados a conocer la conducta de apareamiento y alimentación de los insectos dañadores en el estado adulto, sus depredadores, determinar los mecanismos de selección de hospedante a través, por ejemplo, de ensayos de respuesta antenaria a ciertos compuestos químicos potencialmente atractivos, evaluar el desempeño de larvas en respuesta a la condición de vigor/estrés del hospedante, entre otros. Todas estas posibilidades hacen de este sistema un interesante objeto de estudio en el ámbito de la herbivoría por xilófagos.

\section{AGRADECIMIENTOS}

Agradecemos a Karina Bohle y Andrea Díaz por su apoyo en las actividades de terreno y a Patricio Pliscoff por diseñar la Figura 1. Al proyecto FONDEF D97I1065 "Software de Planificación de Actividades de roble-raulí-coigüe en la Novena y Décima Región", por proveer de la información de inventario, particularmente a Paulo Moreno y Salvador Gezán por proporcionar información que facilitó considerablemente la ubicación de las parcelas. Alicia Ortega, Moisés Osorio, Carlos Ramírez, Claudio C. Ramírez y Ernesto Gianoli contribuyeron a mejorar significativamente versiones previas de este manuscrito al igual que dos revisores anónimos. Este trabajo fue financiado parcialmente por la Dirección de Investigación y Desarrollo de la Universidad Austral de Chile, Proyecto IDS200031 otorgado a A. Aguilar.

\section{LITERATURA CITADA}

AGUILAR A, C CARRASCO, G TRINCADO, G VERGARA, R RÍOS \& A DÍAZ (1997) Evaluación de los insectos barrenadores de madera asociados al tipo forestal roble-raulí-coigüe en la provincia de Valdivia, Décima Región: una aproximación hacia la evaluación de pérdidas. CONAF / CPF SA / UACH. Informe de convenio № 235. 36 pp.

ALTIERI M (1992) Biodiversidad, agroecología y manejo de plagas. Consorcio latinoamericano sobre agroecología y desarrollo. CETAL Ediciones, Valparaíso, Chile. 162 pp.

ANDOW DA (1991) Vegetational diversity and arthropod population response. Annual Review of Entomology 36: 561-586.

BALDINI A \& L PANCEL (eds) (2002) Agentes de daño en el bosque nativo. Editorial Universitaria, Santiago, Chile. 282 pp.

BARATA EN, H MUSTAPARTA, JA PICKETT, LJ WADHAMS \& J ARAUJO (2002) Encoding of host and non-host plant odours by receptor neurones in the eucalyptus woodborer, Phoracantha semipunctata (Col: Cerambycidae). Journal of Comparative Physiology A 188: 121-133.

BYERS JA (1989) Chemical ecology of bark beetles. Experientia 45: 271-283.

CABRERA P (1994) Impacto de Holopterus chilensis Blanch. En renovales de Nothofagus obliqua (Mirb) Oerst. en la provincia de Valdivia: avances hacia la evaluación del daño. Tesis Ingeniería Forestal, Facultad de Ciencias Forestales, Universidad Austral de Chile, Valdivia, Chile. 42 pp.

CAMERON RS \& L PEÑA (1982) Cerambycidae associated with the host genus Nothofagus in Chile and Argentina. Turrialba (Argentina) 32: 481-487.

CAMERON RS \& P REAL (1974) Contribución a la biología del "coleóptero de la luma" Cheloderus childreni (Gray) (Coleoptera: Cerambycidae). Revista Chilena de Entomología 8: 123-132.

CARRASCO C (2000) Evaluación del daño y pérdidas de volumen ocasionadas por Cheloderus childreni (Gray), en bosques de Nothofagus dombeyi (Mirb.) Oerst. en la provincia de Valdivia. Tesis Ingeniería Forestal, Facultad de Ciencias Forestales, Universidad Austral de Chile, Valdivia, Chile. 54 pp.

CARRILLO RL \& LM CERDA (1987) Zoofitófagos en Nothofagus chilenos. Bosque (Chile) 8: 99-103.

COLL M \& DG BOTTRELL (1994) Effects of nonhost plants on an insect herbivore in diverse habitats. Ecology 75: 723-731.

DE CAMINO R \& F DRAKE (1977) Estudio comparativo de rodales del género Nothofagus en Chile y Gran Bretaña. Informe Técnico CONAF Novena Región, Temuco, Chile. 28 pp.

DÍAZ A (1999) Evaluación del daño interno y estimación de pérdidas producidas por Holopterus chilensis (Blanch) en Nothofagus obliqua (Mirb.) Oerst. en la provincia de Valdivia, Décima Región. Tesis Ingeniería Forestal, Facultad de Ciencias Forestales, Universidad Austral de Chile, Valdivia, Chile. 71 pp.

DONOSO C (1981) Tipos forestales de los bosques nativos de Chile. Investigación y Desarrollo Forestal. Documento de trabajo № 38. FAO: DP/CH/76/0003. Santiago, Chile. 81 pp.

DONOSO C (1994) Bosques templados de Chile y Argentina. Variación estructura y dinámica. Editorial Universitaria, Santiago, Chile. 484 pp.

DUNN JP, TW KIMMERER \& GL NORDIN (1986) The role of host tree condition in attack of white oaks 
by the twolined chestnut borer, Agrilus bilineatus (Weber) (Coleoptera: Buprestidae). Oecologia 70: 596-600.

DUNN JP, DA POTTER \& TW KIMMERER (1990) Carbohydrates reserves, radial growth, and mechanism of resistance of oak trees to phloemboring insects. Oecologia 83: 458-468.

ELLIOTT HJ, KILE GA, SG CANDY \& DA RATKOWSKY (1987) The incidence and spatial pattern of Nothofagus cunninghamii (Hook.) Oerst, attacked by Platypus subgranosus Scheld in Tasmania's cool temperate rainforest. Australian Journal of Ecology 12: 125-138.

GARA RI, LM CERDA \& E KRAHMER (1978) Sobre la emergencia y vuelo de dos cerambícidos chilenos: Holopterus chilensis y Cheloderus childreni. Turrialba 28: 117-121.

GRANDON F (1996) Análisis fitosanitario de los Nothofagus de Chile, desde el punto de vista entomológico. Tesis Ingeniería Forestal, Facultad de Ciencias Forestales, Universidad Austral de Chile, Valdivia, Chile. 105 pp.

HANKS LM, TD PAINE \& JG MILLAR (1993) Host species preference and larval performance in the wood-boring beetle Phoracantha semipunctata F. Oecologia 95: 22-29.

HANKS LM (1999) Influence of the larval host plant on reproductive strategies of cerambycid beetles. Annual Review of Entomology 44: 483-505.

HUMPHREY JW, C HAWES, AJ PEACE, R FERRISKAAN \& MR JUKES (1999) Relationships between insect diversity and habitat characteristics in plantations forest. Forest Ecology and Management 113: 11-21.

INBAR M, H DOOSTDAR \& RT MAYER (2001) Suitability of stressed and vigorous plants to various insect herbivores. Oikos 94: 228-235.

JACTEL H, M GOULARD, P MENASSIEU \& G GOUJON (2002) Habitat diversity in forest plantaton reduces infestations of the pine stem borer Dioryctria sylvestrella. Journal of Applied Ecology 39: 618-628.

KLEINHENTZ M, H JACTEL \& P MENASSIEU (1999) Terpene attractant candidates of Dioryctria sylvestrella in maritime pine (Pinus pinaster) oleoresin, needles, liber, and headspace samples. Journal of Chemical Ecology 25: 2741-2756.

KRUUSE L (1981) Algunos factores bióticos y abióticos que inciden en el nivel de ataque de Holopterus chilensis Blanch. (Col.: Cerambycidae) en Nothofagus obliqua (Mirb.) Oerst. Tesis Ingeniería Forestal, Facultad de Ciencias Forestales, Universidad Austral de Chile, Valdivia, Chile. 76 pp.

LANDIS DA, SD WRATTEN \& MG GURR (2000) Habitat management to conserve natural enemies of arthropod pests in agriculture. Annual Review of Entomology 45: 175-201.
LAWTON JH (1983) Plant architecture, and the diversity of phytophagous insects. Annual Review of Entomology 28: 23-39.

LINSLEY EG (1959) Ecology of Cerambycidae. Annual Review of Entomology 4: 99-138

MANQUI R (2001) Contribución al conocimiento del daño provocado por Holopterus chilensis (Blanch) en Nothofagus obliqua (Mirb) Oerst. y Cheloderus childreni Gray en Nothofagus dombeyi (Mirb) Oerst. en renovales de la Novena Región de Chile. Tesis Ingeniería Forestal, Facultad de Ciencias Forestales, Universidad Austral de Chile, Valdivia, Chile. 71 pp.

McCANN JM \& DM HARMAN (1990) Influence of the intrastand position of black locust trees on attack rate of the locust borer (Coleoptera: cerambycidae). Annals of the Entomological Society of America 83: 705-711.

PEÑA L (1974) Algunas observaciones sobre especies poco conocidas de cerambycidae (Coleoptera). Parte II. Boletín de la Sociedad de Biología de Concepción (Chile) 47: 303-306.

PRICE PW (1997) Insect ecology. Third edition. John Wiley \& Sons, Inc., New York, New York, USA. 607 pp.

PUENTE M, C DONOSO, R PEÑALOZA \& E MORALES (1979) Estudio de raleos y otras técnicas para el manejo de renovales de raulí y $N$. obliqua. Etapa I. Identificación y caracterización de renovales de raulí y $N$. obliqua. Informe de convenio № 5. Proyecto CONAF/PNUD/FAO-CHI. $128 \mathrm{pp}$

REID ML \& T ROBB (1999) Death of vigorous trees benefits bark beetles. Oecologia 120: 555-562.

ROOT RB (1973) Organization of a plant-arthropod association in simple and diverse habitats: the fauna of collards (Brassica oleracea). Ecological Monographs 43: 95-124.

SAS INSTITUTE (1997) SAS/STAT software: changes and enhancements through release 6.12. SAS Institute, Cary, North Carolina, USA.

TONHASCA A Jr \& DN BYRNE (1994) The effects of crop diversification on herbivorous insect: a metaanalysis approach. Ecological Entomology 19: 239 244.

THOMPSON JN (1988) Evolutionary ecology of the relationship between oviposition preference and performance of offspring in phytophagous insect. Entomologia Experimentalis et Applicata 47: 3-14.

ZHANG QH, JA BYERS \& XD ZHANG (1993) Influence of bark thickness, trunk and height on reproduction of the longhorned beetle, Monochamus sutor (Col., Cerambycidae) in burned larch and pine. Journal of Applied Entomology 115: 145-154.

ZHANG QH, F SCHLYTER \& P ANDERSON (1999) Grean leaf volatiles interrupt pheromone response of spruce bark beetle, ICC typographus. Journal of Chemical Ecology 25: 2847-2861. 